\title{
The Topology of Branching Universes
}

\author{
Gordon McCabe
}

February 5, 2008

\begin{abstract}
The purpose of this paper is to survey the possible topologies of branching space-times, and, in particular, to refute the popular notion in the literature that a branching space-time requires a non-Hausdorff topology.
\end{abstract}

\section{Branching MWI space-times}

Two basic types of branching universe have been suggested in modern mathematical physics: universes which branch in a style befitting the 'many-worlds' interpretation (MWI) of quantum theory; and universes which bifurcate to implement a topology-change cobordism.

This section, concerned with the former type of branching universe, is not intended as a defence or analysis of the many-worlds interpretation. It is not even the intention to argue that the many-worlds interpretation genuinely entails such a branching model of space-time. Rather, the intention is to analyse the topological implications of such a branching model of space-time, and to question some of the assumptions normally found in the literature.

The many-worlds interpretation holds that if a system is prepared into a state which is a 'superposition' $\Psi=\sum_{i} a_{i} v_{i}$ with respect to some quantity $A$ whose eigenvectors provide a basis $\left\{v_{i}\right\}$ of the quantum state space, then when that system undergoes a measurement-like interaction, the universe splits into multiple branches, one for each basis vector $v_{i}$. Each different branch records a definite value for the quantity $A$, corresponding to the eigenvector $v_{i}$.

This branching can be thought of as either a local or global process. If the branching is considered to be a global process, then the branches correspond to multiple regions of four-dimensional space-time which emanate from a common three-dimensional hypersurface, (see Figure 1). Each such branching hypersurface has a non-unique future.

The notion that the entire universe branches in this style every time there is a measurement-like interaction, renders such branching a highly non-local process, and tacitly supposes that there is a unique global time coordinate for the universe. Treating a measurement-like interaction as a point event in spacetime, there will be many spacelike hypersurfaces which pass through that point; the selection of only one of these as the branching hypersurface requires one to 


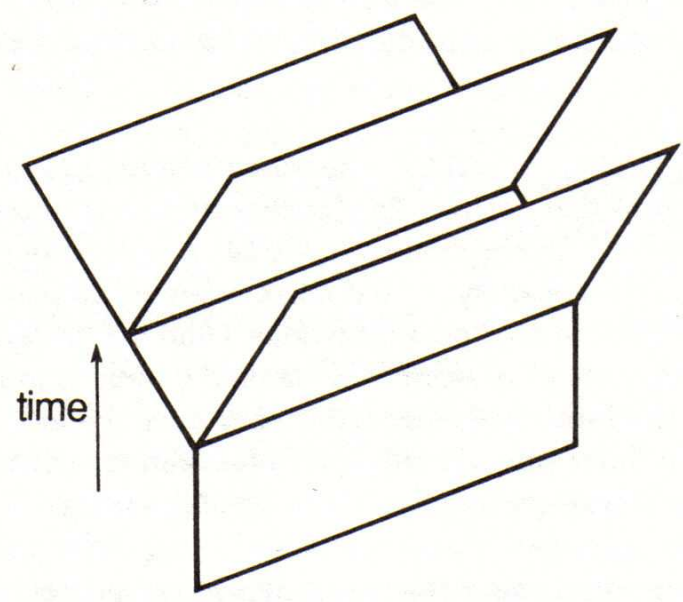

Figure 1: Globally bifurcating space-time, from Earman 1986, p225

accept that there is a preferential time coordinate for the universe. To avoid these difficulties, one can suggest that the universe only branches locally as the result of a measurement-like interaction. To be specific, one can suggest that the future light-cone of the interaction event has multiple branches, one for each possible outcome of the interaction. If one imagines such a universe as a twodimensional sheet, then the image is one in which there are numerous pockets in the sheet, formed by the multiple branches of future light cones. Penrose has drawn just such an image of a branching MWI universe, (see Figure 2).

It has been repeatedly claimed, by different authors, for over two decades, that such a model of space-time entails a non-Hausdorff topology, and therefore cannot be a topological manifold. In 1979 Penrose suggests that such branching space-times "could be legitimate mathematical objects, e.g. four-dimensional Lorentzian manifolds subject to Einstein's field equations (say), but where the Hausdorff condition is dropped," (1979, p594). In 2004, Penrose repeats this assertion, suggesting that "non-Hausdorff manifolds can 'branch'," (2004, p222). In the interim, Matt Visser has echoed Penrose's assertion, stating that one "uses non-Hausdorff manifolds to describe 'train track' geometries where the same present has two or more futures," (2003, p164), and claiming that "a non-Hausdorff manifold has the bizarre property that the dimensionality of the manifold is not necessarily equal to the dimensionality of the coordinate patches. From a physicist's perspective, this idea has been explored somewhat by Penrose," (1993, p12). Lee Smolin, dealing with a 'minimalist wormhole' obtained by identifying a pair of points in a manifold, states that "of course, we loose the Hausdorff property," (1994, p2).

Let us review the pertinent definitions to gain some clarity. A topological manifold is a topological space which is: 


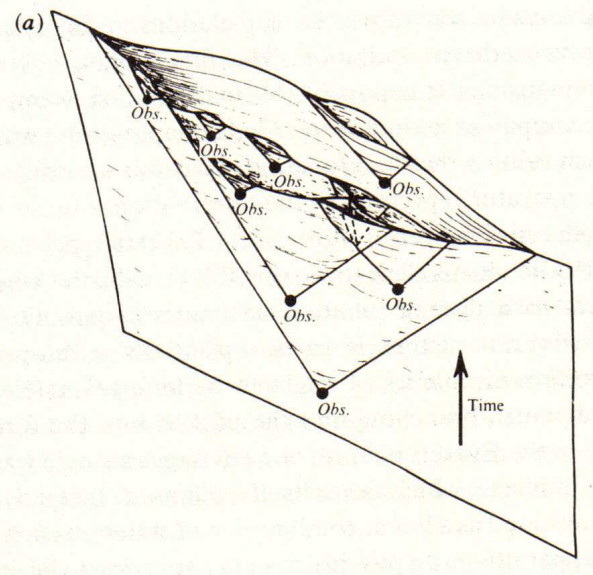

Figure 2: Bifurcating light cones, from Penrose 1979, p593

1. Locally Euclidean. i.e each point $p$ possesses an open neighbourhood $U$ which is homeomorphic with an open subset of some $n$-dimensional Euclidean space $\mathbb{R}^{n}$. The homeomorphism $\phi: U \rightarrow \mathbb{R}^{n}$ is called a coordinate chart, and $n$ is the local dimension.

2. A Hausdorff topological space. i.e each pair of points $p, q$ possess neighbourhoods, $p \in U$ and $q \in V$, which are disjoint from each other, $U \cap V=\emptyset$.

A connected locally Euclidean topological space must be of constant local dimension.

Manifolds of various type, such as smooth manifolds or piecewise-linear manifolds, are topological manifolds which satisfy additional conditions concerning the transformations from one coordinate chart to another, wherever those charts overlap.

Penrose and Visser are suggesting that a branching space-time corresponds to a non-Hausdorff topological space which is still locally Euclidean, of dimension 4, and equipped with a smooth Lorentzian metric. As Visser states, "normally manifolds are assumed Hausdorff by definition. Relaxing this condition permits the existence of "branched' manifolds," (1996, p250). Whilst this is true, one can equally represent branching space-times with topological spaces which are Hausdorff, but not locally Euclidean about every point. Moreover, the diagrams used to represent branching space-times correspond to the latter case, and this fact has been neglected in the literature.

Let us consider a simple example of the distinction between these two types of branching topology. Consider first the example given by Visser of a onedimensional manifold that branches in two:

Begin with the real line $\mathbb{R}$, remove the half-closed interval $I=[0, \infty)$, and replace it with two copies $I_{1}=\left[0_{1}, \infty\right)$ and $I_{2}=\left[0_{2}, \infty\right)$ by taking the union 

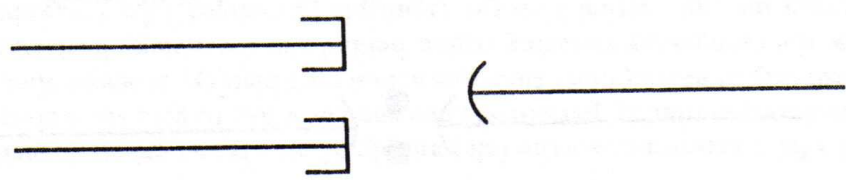

Figure 3: Non-Hausdorff but locally Euclidean, from Visser 1996, p252

$M=(-\infty, 0) \cup\left[0_{1}, \infty\right) \cup\left[0_{2}, \infty\right)$. Define a basis for the topology on $M$ by requiring that any open subset of $(-\infty, 0) \cup\left[0_{1}, \infty\right)$ and any open subset of $(-\infty, 0) \cup\left[0_{2}, \infty\right)$ is an open subset of $M$. This means that the neighbourhood base of $0_{1}$ is provided by the open subsets $(-\epsilon, 0) \cup\left[0_{1}, \epsilon\right)$, for $\epsilon>0$, and the neighbourhood base of $0_{2}$ is provided by the open subsets $(-\epsilon, 0) \cup\left[0_{2}, \epsilon\right)$. As a consequence $0_{1}$ and $0_{2}$ do not possess disjoint neighbourhoods, and the topological space is non-Hausdorff, but still locally Euclidean of dimension 1 about every point. This example of a 1-dimensional non-Hausdorff space is also that given by Hawking and Ellis (1973, p13-14), and Geroch's example of a non-Hausdorff manifold is essentially just a 2-dimensional analogue (1971, p100).

Visser provides two diagrams to represent this non-Hausdorff space. The first diagram, (see Figure 3), clearly represents the fact that $0_{1}$ and $0_{2}$ are distinct points, but the second $(1996, \mathrm{p} 253)$ treats both points as the vertex of a $Y$-shape, and is potentially misleading. Whilst the first diagram is correct, the second, (see Figure 4), is conventionally taken to represent the following Hausdorff space, in which $0_{1}$ and $0_{2}$ are identified:

Begin with the real line $\mathbb{R}$, remove the half-closed interval $I=[0, \infty)$, and replace it with two copies $I_{1}=\left[0_{1}, \infty\right)$ and $I_{2}=\left[0_{2}, \infty\right)$ by (i) taking the union $M=(-\infty, 0) \cup\left[0_{1}, \infty\right) \cup\left[0_{2}, \infty\right)$; (ii) defining an equivalence relationship $R$ which is such that $0_{1} \sim 0_{2}$; (iii) taking the quotient topological space $Q=M \backslash R$. The quotient space $Q$ consists of the set of equivalence classes in $M$, where the only equivalence class containing more than one point is $[0]=\left\{0_{1}, 0_{2}\right\}$. The projection mapping $\pi: M \rightarrow Q$ is such that $\pi\left(0_{1}\right)=\pi\left(0_{2}\right)=[0]$. The quotient topology is defined to be the largest topology one can bestow on $Q$ which still permits the projection mapping to be continuous. Whilst there are potentially many topologies on $Q$ such that the inverse image $\pi^{-1}(W)$ of every open subset $W \subset Q$ is an open subset of $M$, the quotient topology is the strongest of these. A subset $W \subset Q$ is defined to be open if and only if the inverse image $\pi^{-1}(W)$ is an open subset of $M .^{1}$ This quotient construction gives a branching topological space which genuinely corresponds diagrammatically to a $Y$-shape. The quotient topological space is Hausdorff, but not locally

\footnotetext{
${ }^{1}$ Note, this does not mean that the image of every open subset of $M$ projects onto an open subset of $Q$; there may be open subsets $U \subset M$ which are such that $\pi^{-1}(\pi(U))$ is not an open subset of $M$. The projection mapping is said to be open if every open subset of $M$ does project onto an open subset of $Q$.
} 


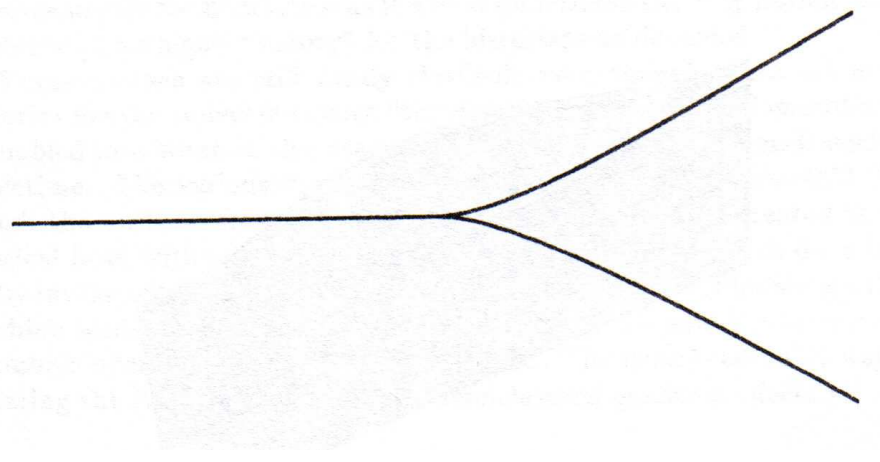

Figure 4: Hausdorff but not locally Euclidean, from Visser 1996, p253

Euclidean about every point. There is only one point associated with the branch, namely [0], and this point can be separated from any other point by disjoint neighbourhoods. However, there is no neighbourhood of this point which can be homeomorphically mapped to an open subset of $\mathbb{R}$. Every neighbourhood of $[0]$ is $Y$-shaped, and such a subset cannot be mapped to an open subset of $\mathbb{R}$ without ripping or tearing.

Every topological space with a metric topology must be a Hausdorff space. The non-Hausdorff space constructed by Visser has a pseudo-metric rather than a metric. i.e there are distinct points, $0_{1}$ and $0_{2}$, which are such that the distance between them $d\left(0_{1}, 0_{2}\right)=0$, violating the positive definite condition for a metric space. The symmetry condition and triangle inequality are preserved, so this space satisfies the conditions of a pseudo-metric space. The fact that $0_{1}$ and $0_{2}$ are separated by zero distance, could be used to justify the diagrammatic representation of both these points by a single vertex to a $Y$-shape. However, such a diagram is ambiguous at best, and conventionally used to represent the case where the points are identified. To obtain a metric space, with Hausdorff topology, from a pseudo-metric space, one merely needs to identify the points with $d(x, y)=0$.

One can generalize these considerations to space-times which branch globally at spacelike hypersurfaces. Taking a 4-dimensional Lorentzian space-time $\mathcal{M}$ which can be foliated as $\mathbb{R} \times \Sigma$, one can remove the region $[0, \infty) \times \Sigma$, and replace it with multiple copies $\left[0_{i}, \infty\right) \times \Sigma$. If the new space is obtained by merely appending each boundary hypersurface $\left\{0_{i} \times \Sigma: i=1, \ldots, n\right\}$ to $(-\infty, 0) \times \Sigma$, the result is a non-Hausdorff space which is locally Euclidean of dimension 4 about every point. In contrast, identifying the corresponding points in each hypersurface $\left\{0_{i} \times \Sigma: i=1, \ldots, n\right\}$ results in a Hausdorff space which fails to be locally Euclidean about every point. Points on the branching hypersurface do not have neighbourhoods which can be homeomorphically mapped to open subsets of $\mathbb{R}^{n}$. Any neighbourhood of a point on the branching hypersurface will include a subset from each one of the four-dimensional regions which emanate from that hypersurface. Such a neighbourhood clearly cannot be mapped to an 
open subset of $\mathbb{R}^{n}$ without tearing the neighbourhood in some way, hence there cannot be a homeomorphism with an open subset of $\mathbb{R}^{n}$. As a consequence, there is no tangent vector space at any of the branch points; one cannot take the sum of vectors which are tangent to different branches emanating from the same point. There is therefore no tensor algebra at those points either. The Einstein field equations, the energy conditions of general relativity, and the expression for the local conservation of energy-momentum, cannot hold at the branching points because these expressions and equations are tensorial. This may be taken as a reason to prefer the non-Hausdorff, locally Euclidean model of branching space-time, but it is a debate which has not been conducted in the literature.

Evaluating the many-worlds interpretation of quantum theory, Earman draws a diagram of such a globally bifurcating space-time, (Figure 1), and states "I do not balk at giving up the notion, held sacred until now, that space-time is a Hausdorff manifold. But I do balk at trying to invent a causal mechanism by which a measurement of the spin of an electron causes a global bifurcation of space-time," (1986, p225). Earman's diagram of a branching space-time is a diagram of a Hausdorff space, which fails to be locally Euclidean at all points, so his willingness to contemplate a non-Hausdorff space-time is rather unnecessary. Moreover, as already suggested by Penrose, the many-worlds interpretation can be taken to imply not a global bifurcation of space-time, but a local branching of future light cones.

To define a space-time which branches not globally, but locally at a point, one begins with a 4-dimensional Lorentzian manifold $\mathcal{M}$, one removes the closure of the causal future $J^{+}(x)$ of a point $x$, and then one replaces it with any number $n$ of copies of $\overline{J^{+}(x)}$. One forms the union $\left[M-\overline{J^{+}(x)}\right] \cup\left[\cup_{i=1}^{n} \overline{J_{i}^{+}(x)}\right]$. To define a non-Hausdorff space, one can take this union, and define its topology to be generated by the open subsets of $\left[M-\overline{J^{+}(x)}\right] \cup \overline{J_{i}^{+}(x)}$, for $i=1, \ldots, n$. The topological space that results is non-Hausdorff, but locally Euclidean of dimension 4 about every point, and has the advantage, therefore, that the Einstein field equations can still be applied at every point. Alternatively, one can define a quotient construction which identifies the corresponding boundary points on each copy of $\overline{J^{+}(x)}$. This results in a Hausdorff topological space which fails to be locally Euclidean about the equivalence classes which consist of more than one point.

Both constructions can be legitimately said to represent branching universes, but the diagrams drawn by Penrose (1979, p593) and Visser (1996, p254) to represent these branching future light cones correspond to the quotient constructions, not the non-Haudorff spaces which these authors claim to be the consequence of branching space-time.

There is certainly no guarantee that the quotient of a Hausdorff space will itself be Hausdorff, but equally, it is false to assume that any quotient will be non-Hausdorff. In fact, if one defines a quotient space by the action of a group upon the original Hausdorff space, and if the action is properly discontinuous, ${ }^{2}$

\footnotetext{
${ }^{2}$ A group $G$ has a properly discontinuous action on a set $M$ if, for any compact subset
} 
then the quotient must be Hausdorff. In general, if one treats the equivalence relationship $R$ which defines a quotient construction on a manifold $M$ as a subset $R \subset M \times M$, then the quotient will be Hausdorff if the projection mapping is an open map, and if $R$ is a closed subset of $M \times M$.

As demonstrated above, examples of non-Hausdorff spaces are usually obtained by appending extra points to existing sets, and giving those points a neighbourhood base which is shared with a point in the existing set. For example, take the real number line, append a point $\star$ to it, and give $\star$ the neighbourhood base of 0 . The union of the existing topology on $\mathbb{R}$ and the neighbourhood base of $\star$ defines a base for a topology on $\mathbb{R} \cup \star$. In this topology one cannot find a neighbourbood of 0 and a neighbourhood of $\star$ which are disjoint, hence the topology is non-Hausdorff. Sequences which converge to 0 also converge to $\star$ in this topology. It is worthwhile noting, however, that this method of appending a point can alternatively be thought of as a quotient construction; one takes two copies of $\mathbb{R}$, and one identifies every pair of corresponding numbers, except for the two zeroes, one of which is re-named $\star$.

Consider Visser's assertion that in a non-Hausdorff manifold, the dimension of the manifold doesn't necessarily equal the dimension of the coordinate patches. This is slightly curious because it doesn't correspond to the type of branching space-time presented by Visser, which is non-Hausdorff but of constant local Euclidean dimension. However, the alternative type of branching manifold, which is Hausdorff, does correspond to the notion of a stratified topological space, and such a space can be thought of as a collection of manifold pieces of different dimensions.

A stratified topological space $X$ is one in which there is a finite filtration by closed subsets

$$
X=X^{n} \supseteq X^{n-1} \supseteq \cdots \supseteq X^{0} \supseteq \emptyset,
$$

for which the strata are the $X_{i}=X^{i}-X^{i-1}$, each of which is a manifold. In effect, then, a stratified space is a disjoint union of manifolds of different dimension.

A stratification should not be confused with a foliation, which is a way of exhaustively cutting up a manifold into manifold pieces of equal dimension. Also note that whilst every $n$-manifold, for $n \geq 1$, will have submanifolds of smaller dimension, so that every $n$-manifold is a stratified space, there are stratified spaces which are not manifolds. These spaces are made up of manifold pieces, but do not themselves constitute a manifold. The branching hypersurfaces in an MWI space-time are 3-dimensional topological manifolds; in the restricted topology, every point of such a hypersurface has a neighbourhood homeomorphic with an open subset of $\mathbb{R}^{3}$. With the unrestricted topology, the points in a branching hypersurface have only neighbourhoods that extend into the multiple branches that emanate from the hypersurface, and this prevents the unrestricted topological space from being locally Euclidean.

$C \subset M$, the set $\{\phi \in G: \phi(C) \cap C \neq \emptyset\}$ is finite. 


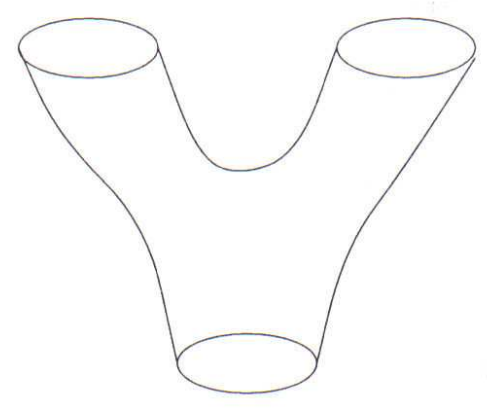

Figure 5: Bifurcating topology-change space-time, from Borde et al 1999, p12

\section{Topology-change branching space-times}

The other type of branching universe, most usually discussed in the context of general relativity and quantum gravity, is a topology-change universe in which the number of connected components in the future boundary is greater than the number of components in the initial boundary. For example, if one starts with the complex projective space $\mathbb{C P}^{2}$, and one removes three copies of the interior of the closed 4-ball $D^{4}$, then one obtains a 4-dimensional manifold with three boundary components homeomorphic to $S^{3}$. This compact 4-manifold is of Euler characteristic zero, hence it can be equipped with a Lorentzian metric tensor. One can further supply this 4-manifold with a time-orientation which renders one copy of $S^{3}$ as the initial boundary, and the other two copies as the final boundary. Such a space-time represents a universe which bifurcates in two, (Friedman 1991, p558). Figure 5 displays a 2-dimensional analogue, often referred to as the 'trousers' space.

Each such branching space-time is a 4-dimensional Lorentzian manifoldwith-boundary $(\mathcal{M}, g)$, which interpolates between the initial boundary $\left(\Sigma_{i}, \gamma_{i}, \phi_{i}\right)$ and final boundary $\left(\Sigma_{f}, \gamma_{f}, \phi_{f}\right)$. The boundary of each $\mathcal{M}$ must consist of the disjoint union of $\Sigma_{i}$ and $\Sigma_{f}$. In addition, the restriction of the Lorentzian metric $g$ to the boundary components must be such that $g \mid \Sigma_{i}=\gamma_{i}$ and $g \mid \Sigma_{f}=\gamma_{f}$. Each interpolating space-time must be equipped with a smooth matter field history $\Phi$, which satisfies the conditions $\Phi \mid \Sigma_{i}=\phi_{i}$ and $\Phi \mid \Sigma_{f}=\phi_{f}$.

The initial 3-manifold $\Sigma_{i}$ need not be homeomorphic with the final 3manifold $\Sigma_{f}$. Hence, the transition from an initial configuration $\left(\Sigma_{i}, \gamma_{i}, \phi_{i}\right)$ to a final configuration $\left(\Sigma_{f}, \gamma_{f}, \phi_{f}\right)$ could be a topology changing transition. In particular, there could be a transition to a topology with a different number of connected components.

The notion of topology change is closely linked with the concept of cobordism. When a pair of $n$-manifolds, $\Sigma_{1}$ and $\Sigma_{2}$, constitute disjoint boundary components of an $n+1$ dimensional manifold, $\Sigma_{1}$ and $\Sigma_{2}$ are said to be cobordant. Any pair of compact 3-manifolds are cobordant, (Lickorish 1963). Not only 
that, but any pair of compact Riemannian 3-manifolds, $\left(\Sigma_{1}, \gamma_{1}\right)$ and $\left(\Sigma_{2}, \gamma_{2}\right)$, are 'Lorentz cobordant', (Reinhart 1963). i.e. There exists a compact 4-dimensional Lorentzian manifold $(\mathcal{M}, g)$, with a boundary $\partial \mathcal{M}$ which is the disjoint union of $\Sigma_{1}$ and $\Sigma_{2}$, and with a Lorentzian metric $g$ that induces $\gamma_{1}$ on $\Sigma_{1}$, and $\gamma_{2}$ on $\Sigma_{2}$.

This cobordism result is vital because it confirms that topology change is possible. Even when $\left(\Sigma_{1}, \gamma_{1}\right)$ and $\left(\Sigma_{2}, \gamma_{2}\right)$ are compact Riemannian 3-manifolds with different topologies, there exists an interpolating space-time.

It is impossible for a 4-dimensional manifold bounded by a couple of nonhomeomorphic 3-dimensional manifolds to be foliated by a one-parameter family of 3-dimensional manifolds. However, it is often said that the topology-change space-time can be described by a Morse-function, a mapping $f: \mathcal{M} \rightarrow[0,1]$ which is such that $f \mid \Sigma_{i}=0$ and $f \mid \Sigma_{f}=1$. For each $a \in[0,1]$, the set of points $f^{-1}(a)$ provides a slice through the space-time. For a topology-change spacetime there will be at least one so-called critical slice, which contains isolated critical points at which $\partial_{\mu} f=0$. In terms of the 2 -dimensional trousers spacetime, one can foliate it, beginning at the waist, with a one-parameter family of circles until one reaches the saddle-like branching from which the legs emanate. There is one slice which intersects the base-point of this saddle, and this slice comprises a pair of circles joined at a single point. The foliation then resumes, each slice comprising a pair of disjoint circles. The slice comprised of a pair of circles joined at a single point is the critical slice, and the joining point of the circles is the critical point.

By Geroch's well-known theorem (1967), if one supplies a time-orientation that renders one hypersurface $\Sigma_{i}$ as the initial hypersurface, and the other hypersurface $\Sigma_{f}$ as the final hypersurface, then the topology-change space-time will necessarily contain closed timelike curves if one adheres to the requirement that the metric tensor must be everywhere Lorentzian and non-degenerate. One can avoid closed timelike curves if one permits the metric to be degenerate, and, in particular, to vanish at isolated points, (Dowker 2003).

If one retains the requirement that the metric tensor be well-defined and non-degenerate everywhere, then the metric tensor will be well-defined and non-degenerate at every point of the topology-change four-manifold $\mathcal{M}$, including the isolated critical points of the Morse function. However, in terms of a one-parameter slicing of $\mathcal{M}$, where each slice is equipped with the induced 3-dimensional geometry, the 3-dimensional metric tensor is not well-defined at the critical points. This is not the same as saying that the 4-dimensional metric tensor is degenerate at these points. Because a critical slice is not locally Euclidean at the critical points, and therefore not a topological manifold, the induced 3-dimensional metric cannot be expressed at the critical points. The critical slices are Hausdorff, but fail to be locally Euclidean at all points. In the simple trousers space, the point where two circles are joined is topologically the centre of a cross, and no cross-like neighbourhood can be mapped homeomorphically to an open subset of $\mathbb{R}$.

This is an opposite type of failure to that suffered by the branching MWI space-times. The latter fail to be topological 4-manifolds, but the branching 
hypersurfaces are topological 3-manifolds. In contrast, a topology-change spacetime is a topological 4-manifold, but it has critical slices which are not topological 3-manifolds.

\section{References}

[1] Borde, A., Dowker, H.F., Garcia, R.S., Sorkin, R.D., Surya, S. (1999). Causal continuity in degenerate spacetimes. arXiv:gr-qc/9901063

[2] Dowker, F. (2003). Topology change in quantum gravity, in The Future of Theoretical Physics and Cosmology, edited by G.W.Gibbons, E.P.S. Shellard and S.J.Rankin, pp436-452. Cambridge: Cambridge University Press.

[3] Earman, J. (1986). A Primer on determinism. Dordrecht, Holland: Reidel.

[4] Friedman, J.L. (1991). Space-time topology and quantum gravity. In A.Ashtekar and J.Stachel (eds.), Conceptual Problems of Quantum Gravity, (pp539-569), Boston: Birkhauser.

[5] Geroch, R.P. (1967). Topology in general relativity. Journal of Mathematical Physics, 8, pp782-786.

[6] Geroch, R.P. (1971). Space-time structure from a global viewpoint, in R.K. Sachs (ed.), General Relativity and Cosmology, Enrico Fermi School Course XLVII, pp71-103. New York: Academic Press.

[7] Hawking, S.W., Ellis, G.F.R. (1973). The large-scale structure of spacetime. Cambridge: Cambridge University Press.

[8] Lickorish, W.B.R. (1963). Homeomorphisms of non-orientable twomanifolds. Proceedings of the Cambridge Philosophical Society, 59, pp307317.

[9] Penrose, R. (1979). Singularities and time asymmetry, in General Relativity: An Einstein Centenary Survey, edited by S. W. Hawking and W. Israel, pp581-638, Cambridge University Press: Cambridge.

[10] Penrose, R. (2004) The Road to Reality, London: Jonathan Cape.

[11] Reinhart, B.L. (1963). Cobordism and the Euler number. Topology, 2, pp173-177.

[12] Smolin, L. (1994). Fermions and Toplogy. arxiv:gr-qc/9404010

[13] Visser, M. (1993). From wormhole to time machine: Remarks on Hawking's chronology protection conjecture, Physical Review, D47, 554-565.

[14] Visser, M. (1996). Lorentzian wormholes: From Einstein to Hawking. AIP Press. New York: Springer Verlag. 
[15] Visser, M. (2003). The quantum physics of chronology protection, in The Future of Theoretical Physics and Cosmology, edited by G.W.Gibbons, E.P.S. Shellard and S.J.Rankin, pp161-176. Cambridge: Cambridge University Press. 